\title{
FAMILIAL SPONTANEOUS PNEUMOTHORAX.
}

\author{
$B y$ F. E. SAXBY WILLIS, M.C., M.D., M.R.C.P.
}

(Physician, Royal Chest Hospital, Hampstead General Hospital, Weir Hospital, and Metropolitan Ear, Nose, and Throat Hospital.)

Cases of spontaneous pneumothorax sometimes occur in apparently healthy individuals. Such cases have been variously termed "Idiopathetic" or "Pneumothorax Simplex" (Kjaergaard). It is a spontaneous pneumothorax which appears in perfectly healthy young persons in whom neither tuberculous nor other inflammatory disease can be demonstrated. It takes a benign course, without exudate, and has a tendency to spontaneous recovery.

Kjaergaard ("Spontaneous Pneumothorax in the Apparently Healthy," I932) gives it as his opinion that the disease is due to the rupture of a valve vesicle on the surface of the lung, which may be of two varieties. One form which he terms the "scar vesicle" develops especially in the apices of the lungs, next to the scar which may originate from a small healed tuberculous focus. These vesicles may be multiple.

The other form is produced through local emphysematous changes, without scar tissue, by the rupture of a "valve vesicle." The latter may be found aso large solitary vesicles on the margins of the lungs, or may be multiple. Obviously, in the latter case, there is a tendency to recurrences and cases can be quotede where several attacks have occurred, sometimes at long intervals. The largest number of recurrences in the present writer's experience, has been I2.

The cases described here are in two members of the same family, brother and sister.

The literature as collected by Kjaergaard contained only three reports of the familial occurrence of spontaneous pneumothorax. He regards these familial cases as supporting the thesis that there is a congenital tendency to the formation of valve vesicles.

\section{Case I.}

On January 29th 1934, Miss W.S.F. came under my observation complaining of recent pain in the left side of her chest with sudden dyspnœa. This had been preceded a few days before, by a feverish cold with cough. Her age was 18 , and she was a telephonist by occupation.

On examining her I found that there were absent breath sounds in the upper part of the left side of the chest. X-ray examination showed a slight degree of collapse at the apex on the left side, due to a small pneumothorax. She made a rapid recovery and by February 14th, the lung had re-expanded and the patient was free from symptoms, and able to leave hospital.

She was not seen again by me until October 1936, when she stated that she had recently had another attack of pain in the left side of her chest with breathlessness 
which quickly passed off, and she now complained of pain in the upper part of the right side of her chest. She was not in any respiratory distress, and walked to the hospital.

On examination, the temperature was $99^{\circ}$, pulse rate 116 , and her respiration 24. The heart was not displaced, breath sounds were audible over both lungs, but there were some scattered fine crepitant râles over the left lung posteriorly. X-ray examination showed a small bilateral pneumothorax confined to the apical regions. She did not wish to remain in hospital, but rested at home for three weeks. At the end of this time a further skiagraph was taken which showed complete re-expansion of both lungs. She expressed herself as feeling quite well and returned to work shortly afterwards, and has remained well since.

\section{Case II.}

On the 28th September 1934, the brother of the above patient and a draughtsman by occupation, aged 20 , attended hospital complaining of pain in the left side of the chest, and slight dyspnœa. Four days previously he had developed a cold with slight fever and an irritating cough with expectoration. The onset of the pain was sudden, after coughing.

On examination his temperature was $99.4^{\circ}$, pulse rate 100 , respirations 22 . There was no cardiac displacement.

He was admitted to the Royal Chest Hospital where examination of the chest showed little that was abnormal apart from some weakness of breath sounds over the left lung, and some scattered crepitations. The X-ray examination revealed the presence of a small pneumothorax in the left apical region. Sputum examination for tubercle bacilli was consistently negative. The sedimentation rate was normal.

He made an uneventful recovery, and was able to leave hospital in three weeks' time. 